\title{
Modulation of Wolframin Expression in Human Placenta during Pregnancy: Comparison among Physiological and Pathological States
}

\author{
Angela Lucariello, ${ }^{1}$ Angelica Perna, ${ }^{1}$ Carmine Sellitto, ${ }^{1}$ Alfonso Baldi, ${ }^{2}$ \\ Alessandro Iannaccone, ${ }^{3}$ Luigi Cobellis, ${ }^{4}$ Antonio De Luca, ${ }^{1}$ and Maria De Falco ${ }^{5,6}$ \\ ${ }^{1}$ Department of Mental and Physical Health and Preventive Medicine, Section of Human Anatomy, Second University of Naples, \\ Largo Madonna delle Grazie, 80138 Naples, Italy \\ ${ }^{2}$ Department of Sciences and Environmental, Biological and Pharmaceutical Technologies, Second University of Naples, \\ Via Vivaldi 43, 81100 Caserta, Italy \\ ${ }^{3}$ Hamilton Eye Institute, University of Tennessee Health Science Center, 930 Madison Avenue, Memphis, TN 38163, USA \\ ${ }^{4}$ Department of Gynaecology, Obstetric and Reproductive Science, Second University of Naples, Via De Crecchio 4, 80138 Naples, Italy \\ ${ }^{5}$ Department of Biology, Section of Evolutionary and Comparative Biology, University of Naples "Federico II", \\ Via Mezzocannone 8, 80134 Naples, Italy \\ ${ }^{6}$ National Institute of Biostructures and Biosystems (INBB), Viale medaglie d'Oro 305, 00136 Rome, Italy
}

Correspondence should be addressed to Maria De Falco; madefalco@unina.it

Received 29 April 2013; Revised 24 October 2013; Accepted 6 November 2013; Published 23 January 2014

Academic Editor: Heather F. Smith

Copyright (C) 2014 Angela Lucariello et al. This is an open access article distributed under the Creative Commons Attribution License, which permits unrestricted use, distribution, and reproduction in any medium, provided the original work is properly cited.

The WFS1 gene, encoding a transmembrane glycoprotein of the endoplasmic reticulum called wolframin, is mutated in Wolfram syndrome, an autosomal recessive disorder defined by the association of diabetes mellitus, optic atrophy, and further organ abnormalities. Disruption of the WFS1 gene in mice causes progressive $\beta$-cell loss in the pancreas and impaired stimulus-secretion coupling in insulin secretion. However, little is known about the physiological functions of this protein. We investigated the immunohistochemical expression of wolframin in human placenta throughout pregnancy in normal women and diabetic pregnant women. In normal placenta, there was a modulation of wolframin throughout pregnancy with a strong level of expression during the first trimester and a moderate level in the third trimester of gestation. In diabetic women, wolframin expression was strongly reduced in the third trimester of gestation. The pattern of expression of wolframin in normal placenta suggests that this protein may be required to sustain normal rates of cytotrophoblast cell proliferation during the first trimester of gestation. The decrease in wolframin expression in diabetic placenta suggests that this protein may participate in maintaining the physiologic glucose homeostasis in this organ.

\section{Introduction}

Wolfram syndrome, also known as DIDMOAD (diabetes insipidus, diabetes mellitus, optic atrophy, and deafness), is an autosomal recessive disorder [1] caused by more than 100 mutations in the WFS1 gene, which was identified by positional cloning in 1998 [2-4]. Most are inactivating mutations, suggesting that loss of function may be responsible for the disease phenotype $[5,6]$. Wolfram patients demonstrate noninflammatory atrophic changes in the brain [7] and in pancreatic islets, resulting in progressive diabetes, blindness, deafness, and other severe neurological defects $[8,9]$. Consistent with these data, it has been demonstrated that the WFS1 gene is expressed at very high levels in the brain and in pancreatic islets $[10,11]$.

In humans, wolframin expression was distributed in many organs, with different tissue and cell localization and expression levels [12]. In foetal systems, wolframin expression 
started faint and increased when development proceeded. In adult human tissues, a variable positive staining was observed in both simple and stratified epithelia [12].

Wolframin is a hydrophobic protein consisting of 890 amino acids with a molecular mass of $\sim 100 \mathrm{kDa}$. This protein is a type II membrane protein with nine putative transmembrane segments [13] and large hydrophilic regions at both termini. Wolframin localizes primarily at the endoplasmic reticulum (ER) in a $\mathrm{N}_{\text {cyt }} / \mathrm{C}_{\text {lum }}$ membrane topology $[3,4,10,14,15]$. Specifically, wolframin colocalized with the ER marker protein disulphide isomerase (PDI) in unstimulated pancreatic $\beta$-cells [4]. Moreover, it has been demonstrated that glucose causes a wolframin translocation from the ER to the Golgi and stimulates the accumulation of wolframin on the plasma membrane, where it forms a complex with AC8-calmodulin and stimulates insulin biosynthesis and secretion [4].

Recently, it has been demonstrated that mice with a disrupted WFS1 gene exhibited impaired glucose homeostasis and a selective $\beta$-cell loss [16]. In WFS1-deficient cells, glucose-stimulated elevation of the cytosolic $\mathrm{Ca}^{2+}$ concentration $\left(\left[\mathrm{Ca}^{2+}\right]_{\text {cyt }}\right)$ was impaired [6], and the ER-stress response was persistently activated [15, 17]. In addition, a recent report suggested that expression of wolframin in oocytes was associated with an increase in $\left[\mathrm{Ca}^{2+}\right]_{\text {cyt }}$ and induced novel cation-selective channel activities in the ER membrane [9, 14]. These data suggested wolframin to play a role in cellular $\mathrm{Ca}^{2+}$ homeostasis and regulation of ER functions [15].

The ER, which constitutes the main intracellular $\mathrm{Ca}^{2+}$ storage area, plays a central role in $\mathrm{Ca}^{2+}$ homeostasis [15]. Various physiological and pathological conditions interfere with these functions, and overloading of these functions induces ER stress. Cells respond to such stress by activating several adaptive pathways, including induction of chaperones, attenuation of protein translation, and apoptosis, collectively called the unfolded protein response, or UPR [18]. Based on the ER localization of wolframin, it is reasonable to speculate that it may play a not yet defined role in the ER stress-induced cell death [14].

Apoptosis plays an important role in formation of human placenta [19-21]. Several investigators have demonstrated that the apoptotic rate increases progressively during normal gestation, which has been interpreted as part of normal placental development [22-25]. The formation of complexes between anti- and proapoptotic proteins appears to regulate cellular sensitivity to apoptosis [24, 26-29]. An abnormal level of apoptosis has been also correlated with a great variety of gestational pathologies such as miscarriages, ectopic pregnancy, intrauterine growth retardation, postterm pregnancy, preeclampsia, and diabetes [21-23, 30, 31].

Based on these premises, we set forth to investigate the expression of wolframin in human placenta throughout gestation in physiological conditions and contrasted it with the expression patterns in diabetes mellitus, a central characteristic of the Wolfram phenotype.

\section{Materials and Methods}

2.1. Samples. Human placental samples were obtained with informed consent from first trimester voluntary termination of gestation (VTG, $n=15$ ), first trimester loss of pregnancy (LP, $n=15)$, following birth from a vaginal delivery (VD, $n=$ 15 ), from caesarean sections (CS, $n=15$ ), and from caesarean sections of patients with diabetes mellitus (DMP, $n=15$ ). The gestation period ranged from 5 to 40 weeks. The specimens were immediately fixed in formalin for immunohistochemistry.

2.2. Immunohistochemistry. Immunohistochemistry was carried out essentially as described previously [32,33]. Briefly, all sections were deparaffinized in xylene, rehydrated through a graded alcohol series, and washed in phosphate-buffered saline (PBS). PBS was used for all subsequent washes and for antiserum dilution. Tissue sections were quenched sequentially in 3\% hydrogen peroxide and blocked with PBS-6\% nonfat dry milk (Biorad) for $1 \mathrm{hr}$ at room temperature. Slides then were incubated at $4^{\circ} \mathrm{C}$ overnight with an affinitypurified rabbit polyclonal immune serum raised against wolframin (803-240; developed by the Lesperance lab) at a 1:100 dilution and then with diluted anti-rabbit biotinylated antibody (Vector Laboratories) for $1 \mathrm{hr}$. All the slides then were processed by the ABC method (Vector Laboratories) for $30 \mathrm{~min}$ at room temperature. Novared (Vector Laboratories) was used as the final chromogen and hematoxylin was used as the nuclear counterstain. Negative controls for each tissue section were prepared by substituting the primary antiserum with the respective preimmune serum. All samples were processed under the same conditions. The expression level of wolframin-stained cells per field $(250 \mathrm{x})$ at light microscopy was calculated and compared in different specimens by two separate observers (A.B. and F.B.) in a double blind fashion and described as absent $(\bigcirc)$, very low $(\boldsymbol{O})$, low $(\mathbf{\bullet})$, moderate (๑), high (००), and very high (००००).

For each specimen, an HSCORE value was derived by summing the percentages of cells/areas stained at each intensity and multiplying that by the weighted intensity of the staining. An average of 22 fields was observed for each tissue by three observers at different times and the average score was used. All values were expressed as mean \pm standard error of mean (SEM) and differences were compared by one-way analysis of variance (ANOVA) with SAS statistical software (SAS Institute, Cary, NC, USA). $P$ values less than 0.05 were considered significant.

\section{Results}

By immunohistochemical criteria, we observed a modulation of human placental wolframin expression from the first to the third trimester of gestation. Specifically, we showed a high wolframin expression in cytotrophoblast cells, the inner proliferative layer during the first trimester of gestation (Figure 1). Wolframin expression decreased to a moderate level of expression in the third trimester of gestation, at which time it mainly localized in the stroma and in the endothelial cells of placental villi (Figure 2). In diabetic women, the decrease in wolframin placental expression observed in the third trimester of gestation was greater than in healthy women, diminishing from a moderate to a low expression level (Figure 2). 


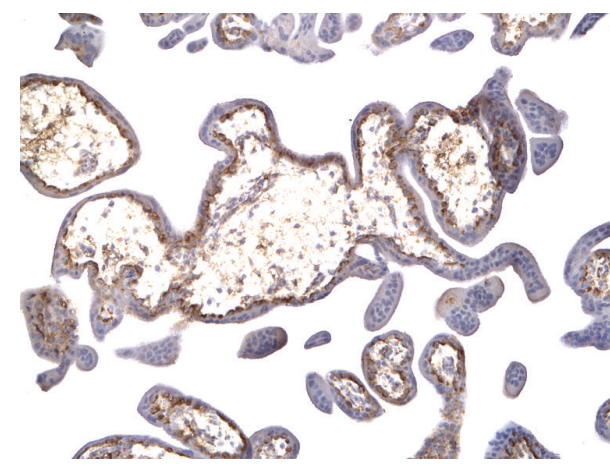

(a)

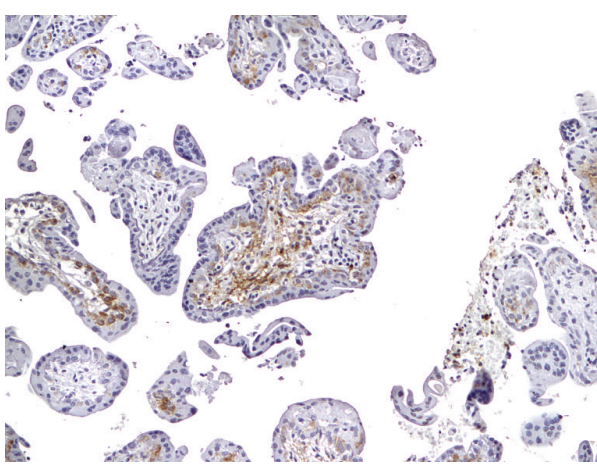

(c)

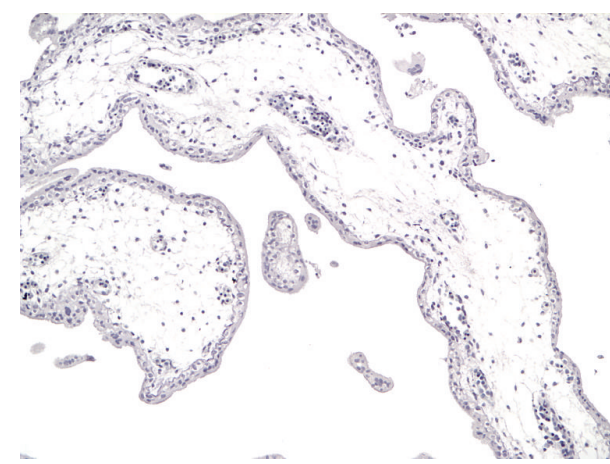

(e)

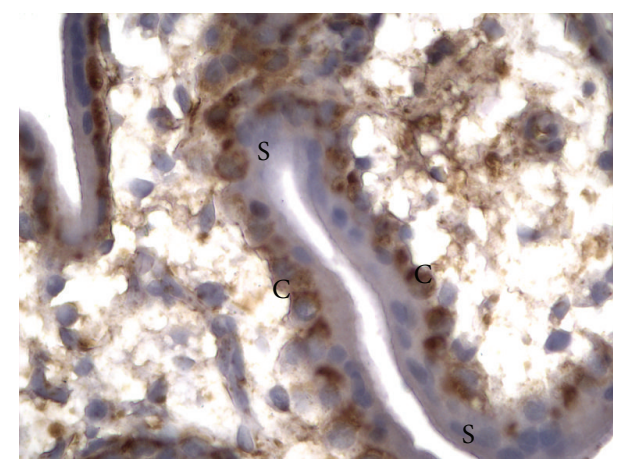

(b)

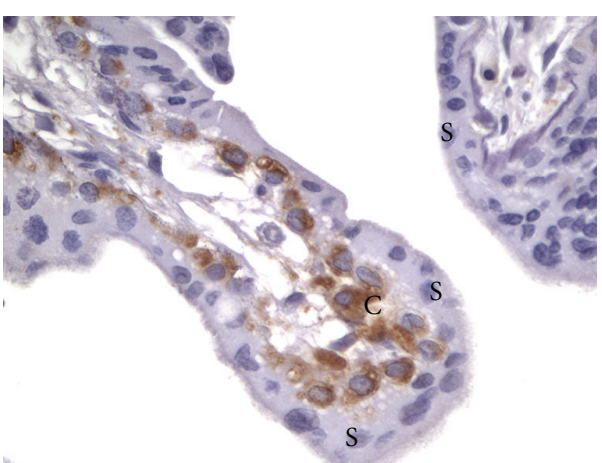

(d)

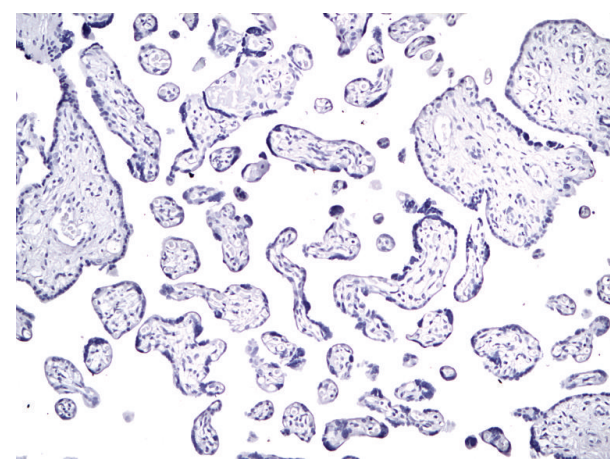

(f)

FiguRE 1: (a) Very high wolframin expression distributed in the cytotrophoblast cells of placental villi from the first trimester voluntary termination of gestation (VTG), 150x; (b) higher magnification showing the very high immunostaining for wolframin localized at perinuclear level in the cytotrophoblast cells from VTG, 640x; (c) moderate wolframin expression in the cytotrophoblast cells and in the stroma of placental villi from loss of pregnancy (LP), 150x; (d) higher magnification showing the moderate wolframin immunopositivity in the cytoplasm of cytotrophoblast cells from LP, 640x; (e) representative negative control of the first trimester VTG, 150x; (f) representative negative control of the third trimester vaginal delivery (VD), 150x. For all the images, C represents cytotrophoblast and S represents syncytiotrophoblast.

In addition, we characterized the modulation of wolframin expression in human placenta throughout the gestation (Tables 1 and 2). In the first trimester VTG placentas, we observed a very high wolframin expression in the cytoplasm of the cytotrophoblast cells forming the inner layer of placental villi (Figure 1(a)). In these cells, wolframin was localized at the perinuclear level (Figure 1(b)). Unlike these cells, the syncytiotrophoblast, the outer differentiated layer of placental villi, appeared completely negative for this protein. In the first trimester LP placentas, wolframin expression slightly decreased to a moderate/high level of expression in the cytotrophoblast cells, whereas a moderate/high immunopositivity for this protein was observed in the stroma of placental villi (Figures 1(c) and 1(d)). In VD placentas, we observed a low wolframin expression distributed in the stroma and the endothelial cells lining blood vessels inside placental villi (Figure 2(a)). Low wolframin immunopositivity was also detectable in the cytoplasm of some cytotrophoblast cells (Figure 2(b)). Syncytiotrophoblast appeared to be wolframinnegative. In CS placentas, we observed an increased wolframin expression to a moderate level of expression, with localization mainly at the endothelial cells and to some 


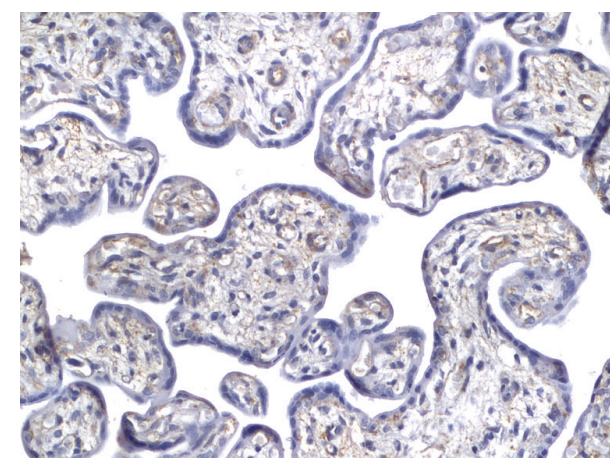

(a)

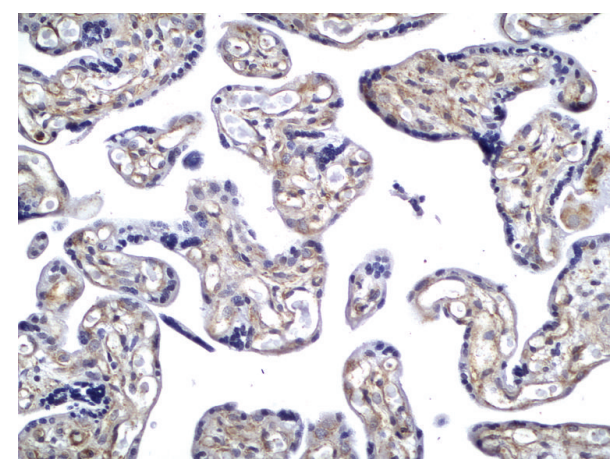

(c)

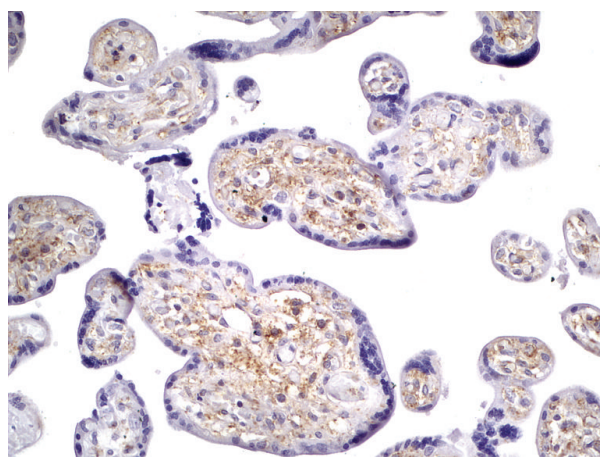

(e)

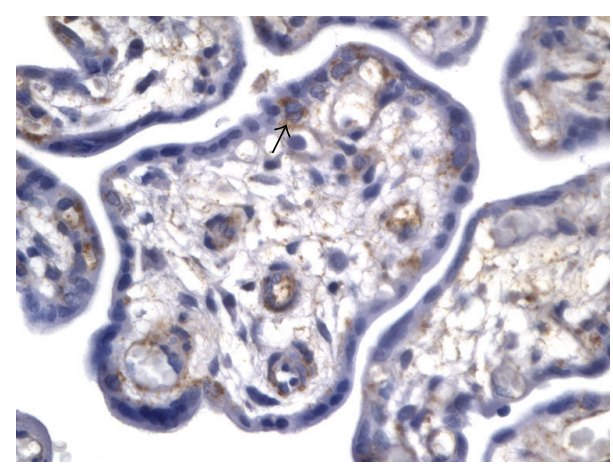

(b)

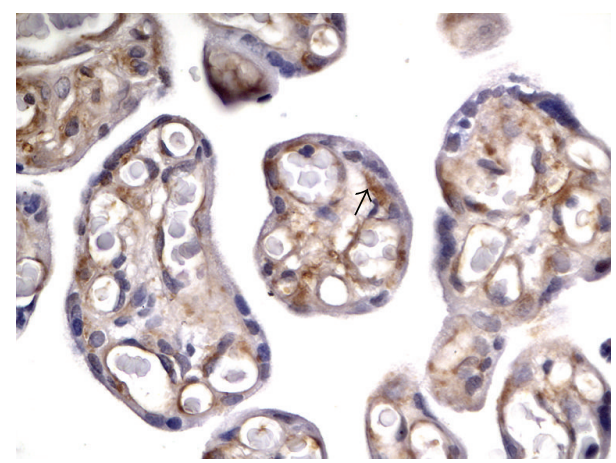

(d)

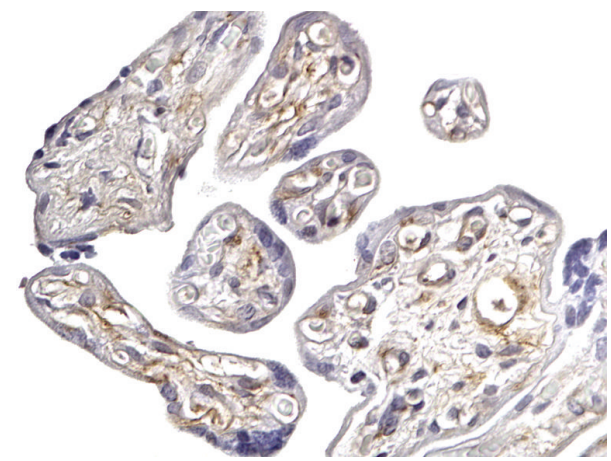

(f)

FIGURE 2: (a) Low wolframin expression in the endothelial cells inside placental villi from the third trimester vaginal delivery (VD), 300x; (b) higher magnification showing wolframin immunopositivity localized in the cytotrophoblast cells (arrow) from the VD, 640x; (c) moderate wolframin immunopositivity in the endothelial cells lining blood vessels of placental villi from caesarean section (CS), 300x; (d) higher magnification showing moderate wolframin expression in endothelial cells and in cytotrophoblast cells (arrow) of placental villi from CS, 640x; (e) low expression level of wolframin in the stroma and endothelial cells of the third trimester placental villi from diabetic women, 300x; (f) higher magnification showing low wolframin expression localized almost exclusively in the stroma and endothelial cells of placenta from diabetic women; no immunopositivity was shown in both cytotrophoblast and syncytiotrophoblast.

cytotrophoblast cells of placental villi (Figures 2(c) and 2(d)). Also in this case, syncytiotrophoblast appeared negative for this protein. On the contrary, in placentas of the third trimester of gestation from DMP women, we observed a decreased wolframin expression to a low expression level in the stroma and in the endothelial cells (Figures 2(e) and 2(f)). Both cytotrophoblast and syncytiotrophoblast cells appeared completely negative for this protein.

Table 2 and Figure 3 illustrate a comparison in the expression patterns of wolframin immunopositivity in normal human placenta versus diabetic gestation, as ascertained by HSCORE and immunohistochemical staining intensity analysis. We observed a very high expression level of wolframin in the cytotrophoblast cells of placentae of the first trimester in first trimester VTG specimens, compared to a low/moderate expression level of wolframin in the cytotrophoblast cells of the third trimester placentae of both VD and CS births. Interestingly, in diabetic women, we did not observe any wolframin expression in the cytotrophoblast cells of the third trimester placental villi. In addition, wolframin expression in 
TABLE 1: Wolframin expression in human placenta during gestation.

\begin{tabular}{|c|c|c|c|c|}
\hline & Cytotrophoblast & Syncytiotrophoblast & Endothelial cells & Stroma \\
\hline Voluntary termination of gestation (VTG) & $\bullet \bullet 00$ & O & $\bullet \bullet$ & $\bullet \bullet$ \\
\hline Loss of pregnancy (LP) & $\bullet 00$ & $\mathrm{O}$ & $\bullet$ & 000 \\
\hline Vaginal delivery (VD) & - & $\mathrm{O}$ & $\bullet$ & $\bullet$ \\
\hline Caesarean sections (CS) & $\bullet$ & $\mathrm{O}$ & $\bullet$ & $\bullet$ \\
\hline Diabetes mellitus complicated patients (DMP) & $\mathrm{O}$ & $\mathrm{O}$ & $\bullet$ & $\bullet$ \\
\hline
\end{tabular}

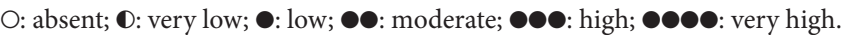

TABLE 2: Wolframin HSCORE values in human placenta during gestation.

\begin{tabular}{lccccc}
\hline & VTG & LP & VD & CS & DMP \\
\hline Cytotrophoblast & $396 \pm 19$ & $212 \pm 14$ & $95 \pm 6$ & $94 \pm 5$ & 0 \\
Stroma & $210 \pm 10$ & $240 \pm 12$ & $98 \pm 7$ & $196 \pm 10$ & $98 \pm 9$ \\
\hline
\end{tabular}

All values were expressed as mean \pm standard error of mean (SEM).

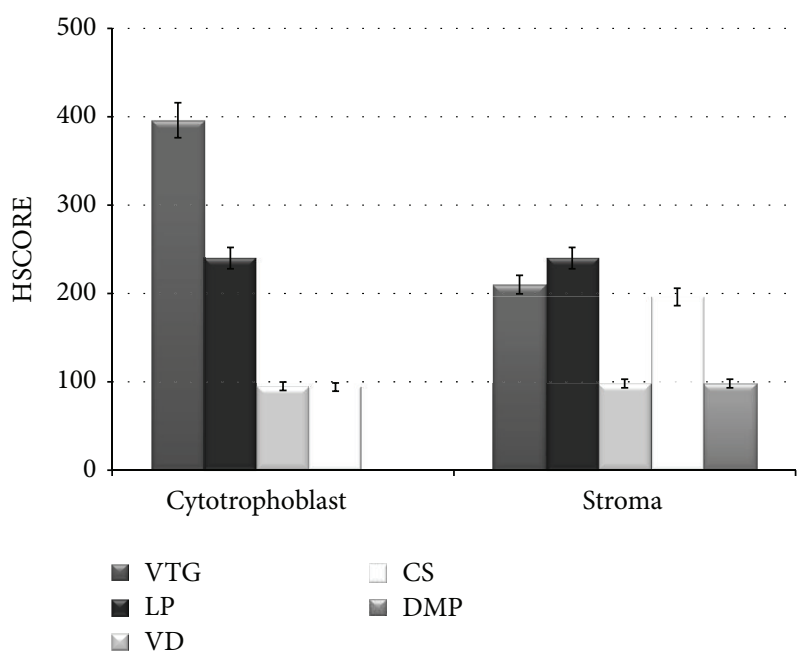

FIGURE 3: Intensity staining of wolframin immunopositivity in human placenta during gestation. Wolframin expression level in placental villi from physiological and pathological human placentas during gestation is shown. Vertical lines show S.E.M. VTG: voluntary termination of gestation, LP: loss of pregnancy, VD: vaginal delivery, CS: caesarean section, and DM: third trimester placentas from women with diabetes mellitus.

the stroma and endothelial cells did not show any significant differences between the first and the third trimester of gestation in both physiological conditions and DMP.

\section{Discussion}

Wolframin acts by regulating cellular $\mathrm{Ca}^{2+}$ homeostasis, at least in part by modulating $\mathrm{ER} \mathrm{Ca}^{2+}$ concentrations [15]. Defective $\mathrm{ER} \mathrm{Ca}^{2+}$ homeostasis, mutations in ER resident proteins, and/or abnormalities of the ER-associated degradation (ERAD) system activate the UPR, in which cells respond by inducing chaperones, attenuating protein translation, and inducing apoptosis [14]. The increase in wolframin expression is attributable, at least in part, to enhanced WFS1 promoter activity stimulated by ER stress-inducing chemicals, thus indicating a direct link between wolframin function and ER stress responses [14]. Therefore, it has been suggested that wolframin plays its physiological role in protecting cells from ER stress-induced apoptosis [14]. Consistent with this hypothesis, it has been demonstrated that WFS1 deficiency increases caspase- 3 cleavage and decreases $\beta$-cell proliferation [17].

Recently, several studies have suggested that apoptosis plays an important role in the normal development, remodeling, and aging of placenta [34]. In addition, it has been demonstrated that apoptosis increases in pregnancies complicated by some disorders such as preeclampsia, foetal growth restriction, and diabetes $[21,30,31,35,36]$. The placenta mediates the nutrition to the growing foetus, provides a barrier from maternal pathogens, and protects the foetus from maternal rejection $[37,38]$. To this end, two different cell populations characterize the villous trophoblast: an inner proliferative layer, the cytotrophoblast, that displays highly proliferative and invasive properties and an outer differentiated layer, the syncytiotrophoblast, displaying various functions necessary to the maintenance of pregnancy and foetal growth with little potential for proliferation [39].

Many molecules are associated with the induction and prevention of apoptosis in different models [21, 31, 40, 41]. Kim et al. [42] and De Falco et al. [19, 24] have demonstrated that the expression of the antiapoptotic factor, Bcl-2, diminishes as gestation progresses, suggesting that a parturitionassociated biological change might induce apoptosis in the placental villi [31]. Recently, we have compared the expression of the proapoptotic Bax protein between first trimester VTGs and first trimester LPs, showing a strong increase of Bax expression in the latter compared to the low/moderate Bax immunopositivity in the former, thereby suggesting that upregulation of apoptosis may be involved in pregnancy complications leading to miscarriage. In addition, we have observed a more intense Bax expression in third trimester VD compared to third trimester CS, which suggests that apoptosis in placental villi may be a key factor in allowing normal delivery [21].

In the present paper, we have first demonstrated that wolframin expression decreases as pregnancy progresses, in accordance with the expression trend of other antiapoptotic factors like Bcl-2 [24]. In addition, we have observed a further decrease in wolframin expression in third trimester placentae from diabetic women, suggesting an important role of this protein in glucose homeostasis also in human placenta. 
Furthermore, we have observed that wolframin expression decreased in LP during the first trimester compared to the intense wolframin expression observed in the first trimester voluntary termination of gestation. This trend of wolframin expression in human placenta was opposite to that of Bax protein [21]. With respect to the third trimester of gestation, we observed an increase of wolframin expression in CS specimens compared to the VD ones, which is again opposite to the pattern of Bax expression [21]. Moreover, we have observed that, in DMP of the third trimester of gestation, wolframin expression strongly decreases in all the placental compartments. Wolframin was reported to negatively regulate activating transcription factor $6 \alpha$, a key transcription factor implicated in ER stress signaling, through the ubiquitinproteasome pathway $[16,43,44]$. This supposed cellular function indicates its important role in cell homeostasis, so decrease of wolframin expression in DMP shows that deregulation processes take place in diabetic women.

\section{Conclusions}

Our results suggest an important role of wolframin in controlling apoptotic events in cytotrophoblast cells of human placenta throughout gestation. These observations can be considered an interesting basis to further investigate subcellular pathways that human placental cells activate in response to physiological and pathological stimuli during gestation.

\section{Conflict of Interests}

The authors have no personal financial or institutional interest for any of the materials described in this paper that might lead to a conflict of interests for any of the authors.

\section{Acknowledgments}

The authors thank Dr. Marci M. Lesperance and Dr. Theresa B. Kim of the Department of Otolaryngology-Head and Neck Surgery, University of Michigan Health System, Ann Arbor, MI, USA, who developed the anti-WFS1 antibody (the generation of which was possible thanks to a K23 Grant no. DC00161 from the National Institute of Deafness and Communication Disorders to Dr. Lesperance and a grant from the American Otological Society, Inc., to Dr. Kim). The authors also gratefully acknowledge the support of intramural funds from the University of Naples "Federico II" (Maria De Falco) and the Second University of Naples (Antonio De Luca and Alfonso Baldi) and of Research to Prevent Blindness, Inc., New York, NY, USA (unrestricted grant to UTHSC Hamilton Eye Institute and a Physician Scientist Award to Alessandro Iannaccone). The authors would like to thank Dr. Pia Furno and Dr. Rosalina Perna for editorial assistance.

\section{References}

[1] D. J. Wolfram and H. P. Wagener, "Diabetes mellitus and simple optic atrophy among siblings: report on four cases," Mayo Clinic Proceedings, vol. 13, pp. 715-718, 1938.
[2] H. Inoue, Y. Tanizawa, J. Wasson et al., "A gene encoding a transmembrane protein is mutated in patients with diabetes mellitus and optic atrophy (Wolfram syndrome)," Nature Genetics, vol. 20, no. 2, pp. 143-148, 1998.

[3] T. M. Strom, K. Hörtnagel, S. Hofmann et al., "Diabetes insipidus, diabetes mellitus, optic atrophy and deafness (DIDMOAD) caused by mutations in a novel gene (wolframin) coding for a predicted transmembrane protein," Human Molecular Genetics, vol. 7, no. 13, pp. 2021-2028, 1998.

[4] S. G. Fonseca, F. Urano, G. C. Weir, J. Gromada, and M. Burcin, "Wolfram syndrome 1 and adenylyl cyclase 8 interact at the plasma membrane to regulate insulin production and secretion," Nature Cell Biology, vol. 14, pp. 1105-1112, 2012.

[5] K. Cryns, T. A. Sivakumaran, J. M. W. Van den Ouweland et al., "Mutational spectrum of the WFS1 gene in Wolfram syndrome, nonsyndromic hearing impairment, diabetes mellitus, and psychiatric disease," Human Mutation, vol. 22, no. 4, pp. 275-287, 2003.

[6] H. Ishihara, S. Takeda, A. Tamura et al., "Disruption of the WFS1 gene in mice causes progressive $\beta$-cell loss and impaired stimulus-secretion coupling in insulin secretion," Human Molecular Genetics, vol. 13, no. 11, pp. 1159-1170, 2004.

[7] P. Shannon, L. Becker, and J. Deck, "Evidence of widespread axonal pathology in Wolfram syndrome," Acta Neuropathologica, vol. 98, no. 3, pp. 304-308, 1999.

[8] D. Genís, A. Dávalos, A. Molins, and I. Ferrer, "Wolfram syndrome: a neuropathological study," Acta Neuropathologica, vol. 93, no. 4, pp. 426-429, 1997.

[9] A. A. Osman, M. Saito, C. Makepeace, M. A. Permutt, P. Schlesinger, and M. Mueckler, "Wolframin expression induces novel ion channel activity in endoplasmic reticulum membranes and increases intracellular calcium," Journal of Biological Chemistry, vol. 278, no. 52, pp. 52755-52762, 2003.

[10] K. Takeda, H. Inoue, Y. Tanizawa et al., "WFS1 (Wolfram syndrome 1) gene product: predominant subcellular localization to endoplasmic reticulum in cultured cells and neuronal expression in rat brain," Human Molecular Genetics, vol. 10, no. 5, pp. 477-484, 2001.

[11] A. C. Riggs, E. Bernal-Mizrachi, M. Ohsugi et al., "Mice conditionally lacking the Wolfram gene in pancreatic islet beta cells exhibit diabetes as a result of enhanced endoplasmic reticulum stress and apoptosis," Diabetologia, vol. 48, no. 11, pp. 2313-2321, 2005.

[12] M. De Falco, L. Manente, A. Lucariello et al., "Localization and distribution of wolframin in human tissues," Frontiers in Bioscience, vol. 4, pp. 1986-1998, 2012.

[13] S. Hofmann, C. Philbrook, K. Gerbitz, and M. F. Bauer, "Wolfram syndrome: structural and functional analyses of mutant and wild-type wolframin, the WFS1 gene product," Human Molecular Genetics, vol. 12, no. 16, pp. 2003-2012, 2003.

[14] K. Ueda, J. Kawano, K. Takeda et al., "Endoplasmic reticulum stress induces WFS1 gene expression in pancreatic $\beta$-cells via transcriptional activation," European Journal of Endocrinology, vol. 153, no. 1, pp. 167-176, 2005.

[15] D. Takei, H. Ishihara, S. Yamaguchi et al., "WFS1 protein modulates the free $\mathrm{Ca} 2+$ concentration in the endoplasmic reticulum," FEBS Letters, vol. 580, no. 24, pp. 5635-5640, 2006.

[16] M. Hatanaka, K. Tanabe, A. Yanai et al., "Wolfram syndrome 1 gene (WFS1) product localizes to secretory granules and determines granule acidification in pancreatic $\beta$-cells," Human Molecular Genetics, vol. 20, no. 7, Article ID ddq568, pp. 12741284, 2011. 
[17] T. Yamada, H. Ishihara, A. Tamura et al., "WFS1-deficiency increases endoplasmic reticulum stress, impairs cell cycle progression and triggers the apoptotic pathway specifically in pancreatic $\beta$-cells," Human Molecular Genetics, vol. 15, no. 10, pp. 1600-1609, 2006.

[18] D. T. Rutkowski and R. J. Kaufman, "A trip to the ER: coping with stress," Trends in Cell Biology, vol. 14, no. 1, pp. 20-28, 2004.

[19] M. De Falco, V. Fedele, L. Cobellis et al., "Immunohistochemical distribution of proteins belonging to the receptor-mediated and the mitochondrial apoptotic pathways in human placenta during gestation," Cell and Tissue Research, vol. 318, no. 3, pp. 599-608, 2004.

[20] M. De Falco, R. Penta, V. Laforgia, L. Cobellis, and A. De Luca, "Apoptosis and human placenta: expression of proteins belonging to different apoptotic pathways during pregnancy," Journal of Experimental and Clinical Cancer Research, vol. 24, no. 1, pp. 25-33, 2005.

[21] L. Cobellis, M. De Falco, M. Torella et al., "Modulation of Bax expression in physiological and pathological human placentas throughout pregnancy," In Vivo, vol. 21, no. 5, pp. 777-784, 2007.

[22] S. C. Smith, P. N. Baker, and E. M. Symonds, "Increased placental apoptosis in intrauterine growth restriction," American Journal of Obstetrics and Gynecology, vol. 177, no. 6, pp. 13951401, 1997.

[23] R. Halperin, S. Peller, M. Rotschild, I. Bukovsky, and D. Schneider, "Placental apoptosis in normal and abnormal pregnancies," Gynecologic and Obstetric Investigation, vol. 50, no. 2, pp. 84-87, 2000.

[24] M. De Falco, L. De Luca, F. Acanfora et al., "Alteration of the Bcl-2: bax ratio in the placenta as pregnancy proceeds," Histochemical Journal, vol. 33, no. 7, pp. 421-425, 2001.

[25] S. L. Straszewski-Chavez, V. M. Abrahams, and G. Mor, "The role of apoptosis in the regulation of trophoblast survival and differentiation during pregnancy," Endocrine Reviews, vol. 26, no. 7, pp. 877-897, 2005.

[26] V. Fulop, S. C. Mok, D. R. Genest, I. Szigetvari, I. Cseh, and R. S. Berkowitz, "c-myc, c-erbb-2, c-fms and bcl-2 Oncoproteins: expression in normal placenta, partial and complete mole, and choriocarcinoma," Journal of Reproductive Medicine for the Obstetrician and Gynecologist, vol. 43, no. 2, pp. 101-110, 1998.

[27] J. Kawiak, G. Hoser, and T. Skórski, "Apoptosis and some of its medical implications," Folia Histochemica et Cytobiologica, vol. 36, no. 3, pp. 99-110, 1998.

[28] L. Danihel, P. Gomolcak, M. Korbel et al., "Expression of proliferation and apoptotic markers in human placenta during pregnancy," Acta Histochemica, vol. 104, no. 4, pp. 335-338, 2002.

[29] D. W. Burleigh, K. Stewart, K. M. Grindle, H. H. Kay, and T. G. Golos, "Influence of maternal diabetes on placental fibroblast growth factor-2 expression, proliferation, and apoptosis," Journal of the Society for Gynecologic Investigation, vol. 11, no. 1, pp. 36-41, 2004.

[30] D. N. Leung, S. C. Smith, K. F. To, D. S. Sahota, and P. N. Baker, "Increased placental apoptosis in pregnancies complicated by preeclampsia," American Journal of Obstetrics and Gynecology, vol. 184, no. 6, pp. 1249-1250, 2001.

[31] F. Sgarbosa, L. F. Barbisan, M. A. M. Brasil et al., "Changes in apoptosis and Bcl-2 expression in human hyperglycemic, term placental trophoblast," Diabetes Research and Clinical Practice, vol. 73, no. 2, pp. 143-149, 2006.
[32] M. De Falco, L. Cobellis, M. Torella et al., "Down-regulation of aquaporin 4 in human placenta throughout pregnancy," In Vivo, vol. 21, no. 5, pp. 813-818, 2007.

[33] L. Cobellis, F. Caprio, E. Trabucco et al., "The pattern of expression of Notch protein members in normal and pathological endometrium," Journal of Anatomy, vol. 213, no. 4, pp. 464-472, 2008.

[34] R. Austgulen, L. Chedwick, C. V. Isaksen, L. Vatten, and C. Craven, "Trophoblast apoptosis in human placenta at term as detected by expression of a cytokeratin 18 degradation product of caspase," Archives of Pathology and Laboratory Medicine, vol. 126, no. 12, pp. 1480-1486, 2002.

[35] J. Y. Han, Y. S. Kim, G. J. Cho et al., "Altered gene expression of caspase-10, death receptor-3 and IGFBP-3 in preeclamptic placentas," Molecules and Cells, vol. 22, no. 2, pp. 168-174, 2006.

[36] B. Huppertz, M. Kadyrov, and J. C. P. Kingdom, "Apoptosis and its role in the trophoblast," American Journal of Obstetrics and Gynecology, vol. 195, no. 1, pp. 29-39, 2006.

[37] D. W. Morrish, E. Linetsky, D. Bhardwaj et al., "Identification by subtractive hybridization of a spectrum of novel and unexpected genes associated with in vitro differentiation of human cytotrophoblast cells," Placenta, vol. 17, no. 7, pp. 431-441, 1996.

[38] D. W. Morrish, J. Dakour, and H. Li, "Life and death in the placenta: new peptides and genes regulating human syncytiotrophoblast and extravillous cytotrophoblast lineage formation and renewal," Current Protein and Peptide Science, vol. 2, no. 3, pp. 245-259, 2001.

[39] N. Ishihara, H. Matsuo, H. Murakoshi, J. B. Laoag-Fernandez, T. Samoto, and T. Maruo, "Increased apoptosis in the syncytiotrophoblast in human term placentas complicated by either preeclampsia or intrauterine growth retardation," American Journal of Obstetrics and Gynecology, vol. 186, no. 1, pp. 158-166, 2002.

[40] K. Lotz, G. Pyrowolakis, and S. Jentsch, "BRUCE, a giant E2/E3 ubiquitin ligase and inhibitor of apoptosis protein of the transGolgi network, is required for normal placenta development and mouse survival," Molecular and Cellular Biology, vol. 24, no. 21, pp. 9339-9350, 2004.

[41] H. Yamauchi, K. Katayama, M. Ueno, K. Uetsuka, H. Nakayama, and K. Doi, "Involvement of p53 in 1- $\beta$-D-arabinofuranosylcytosine-induced trophoblastic cell apoptosis and impaired proliferation in rat placenta," Biology of Reproduction, vol. 70, no. 6, pp. 1762-1767, 2004.

[42] C. J. Kim, Y. J. Choe, B. H. Yoon, C. W. Kim, and J. G. Chi, "Patterns of bcl-2 expression in placenta," Pathology Research and Practice, vol. 191, no. 12, pp. 1239-1244, 1995.

[43] S. G. Fonseca, S. Ishigaki, C. M. Oslowski et al., "Wolfram syndrome 1 gene negatively regulates ER stress signaling in rodent and human cells," Journal of Clinical Investigation, vol. 120, no. 3, pp. 744-755, 2010.

[44] N. Cheurfa, G. M. Brenner, A. F. Reis et al., "Decreased insulin secretion and increased risk of type 2 diabetes associated with allelic variations of the WFS1 gene: the data from epidemiological study on the insulin resistance syndrome (DESIR) prospective study," Diabetologia, vol. 54, no. 3, pp. 554-562, 2011. 

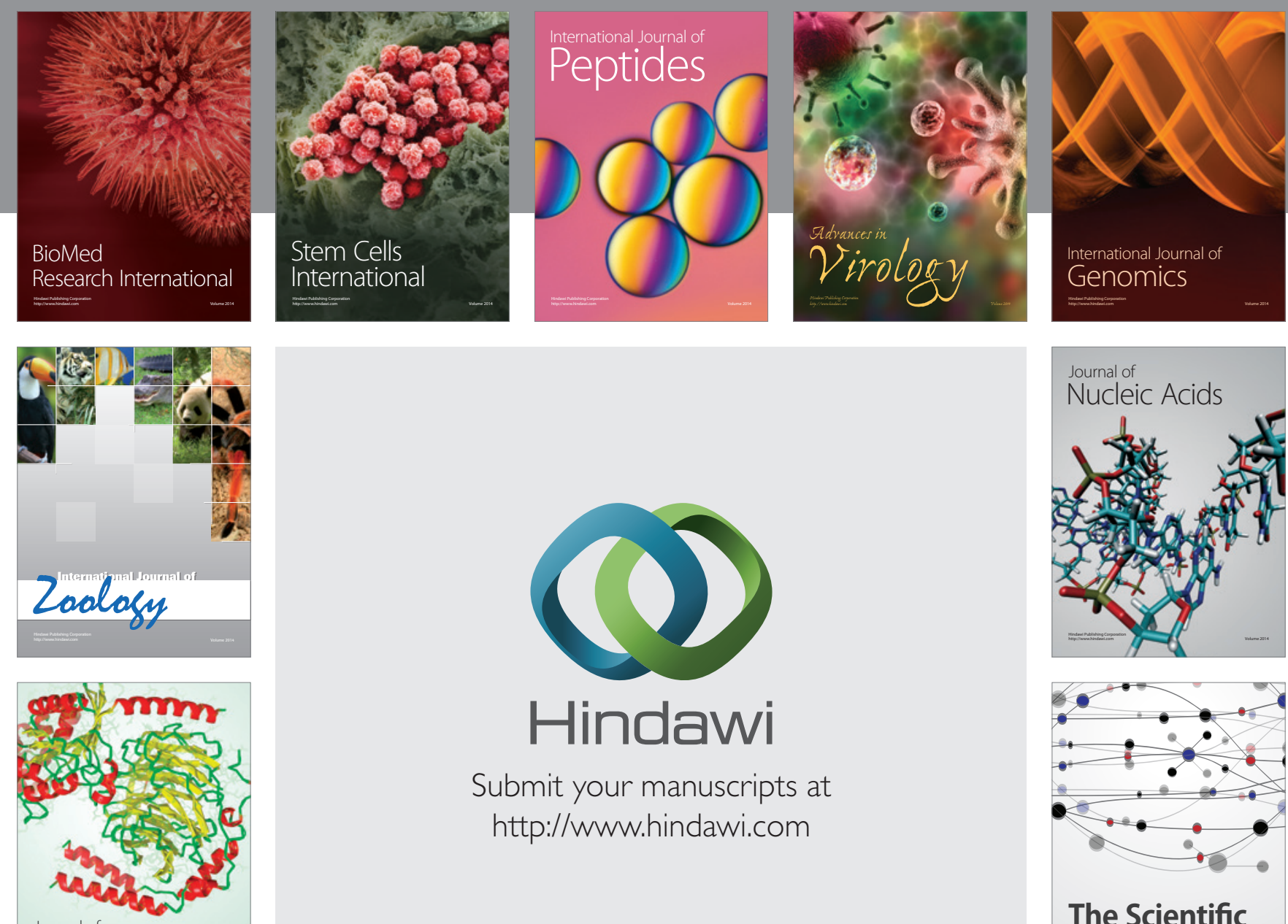

Submit your manuscripts at

http://www.hindawi.com

Journal of
Signal Transduction
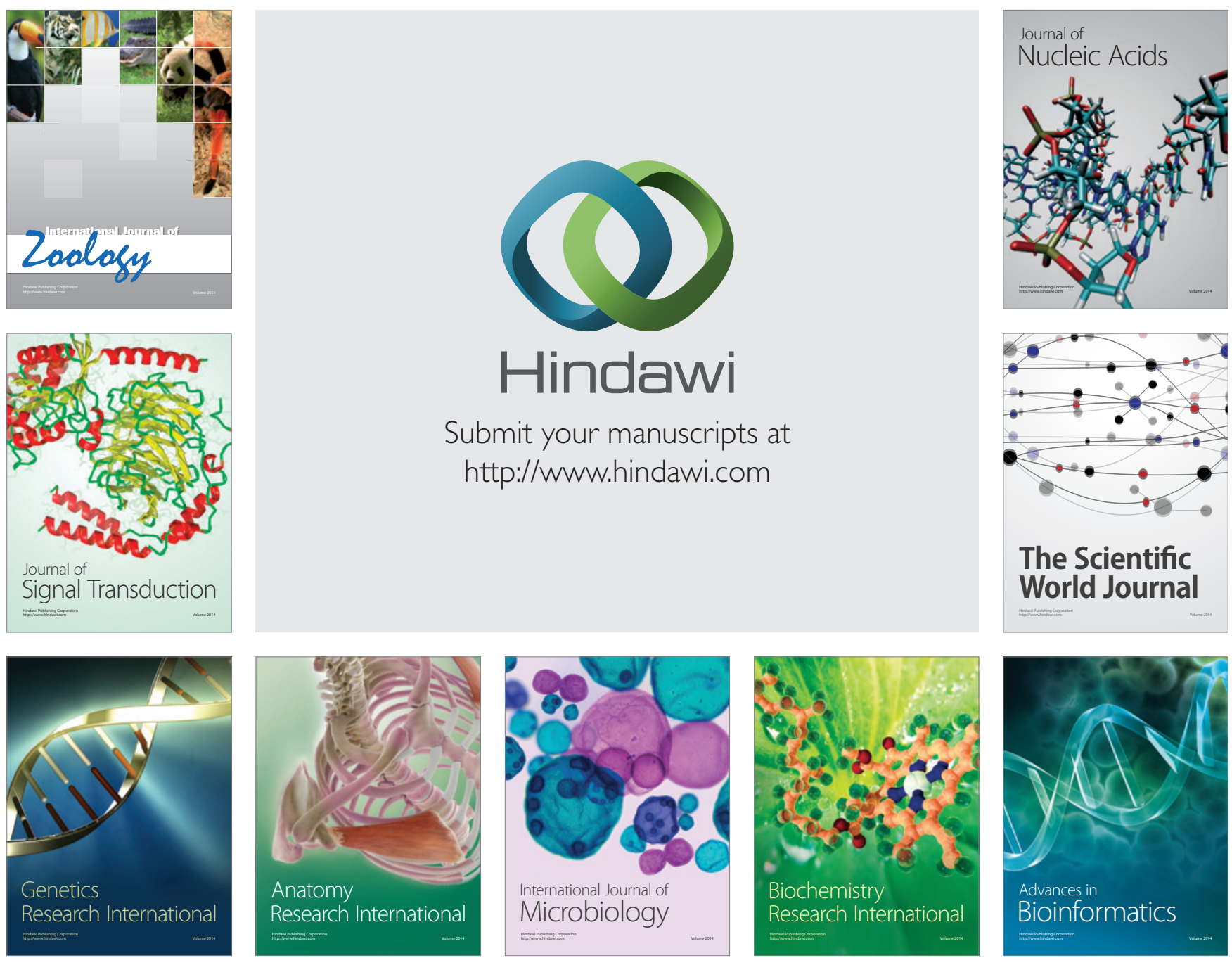

The Scientific World Journal
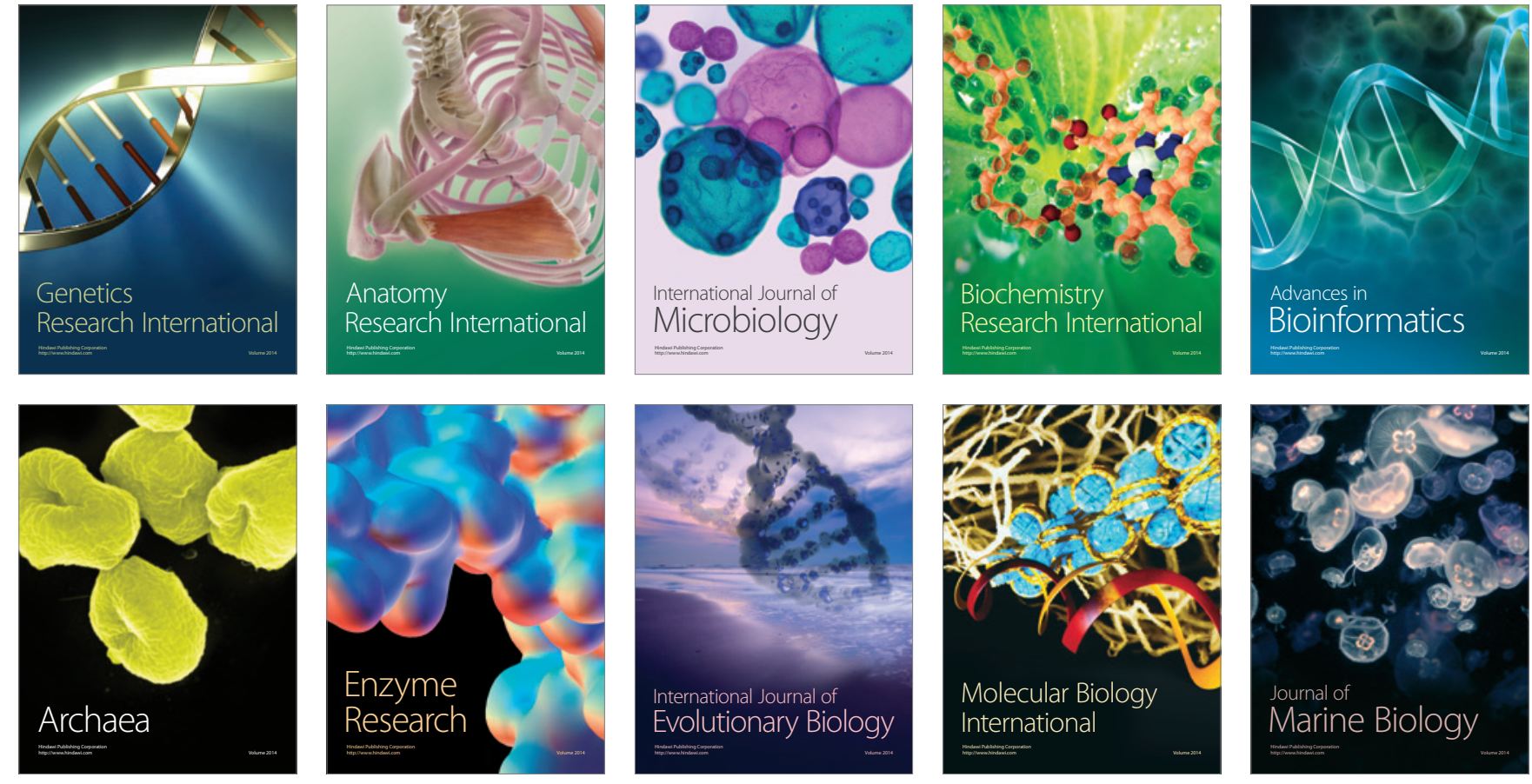